\title{
Efeito da aderência entre camadas na previsão de desempenho de pavimentos asfálticos
}

\section{Anthony Gomes dos Santos ${ }^{1}$, Rosuel Krum Mathias de Assis ${ }^{2}$, José Leomar Fernandes Júnior ${ }^{3}$}

1Universidade de São Paulo, Escola de Engenharia de São Carlos, anthonygomes@usp.br

2Universidade de São Paulo, Escola de Engenharia de São Carlos, rosuel@usp.br

3Universidade de São Paulo, Escola de Engenharia de São Carlos, leomar@sc.usp.br

\section{Recebido:}

22 de fevereiro de 2018

Aceito para publicação:

4 de fevereiro de 2019

Publicado:

31 de agosto de 2019

Editor de área:

Jorge Barbosa Soares

\section{Palavras-chaves:}

Aderência entre camadas,

Resposta estrutural,

Fadiga,

Deformação permanente.

\section{Keywords:}

Adherence between layers,

Structural responses,

Fatigue,

Permanent deformation.

DOI:10.14295/transportes.v27i2.1597

\begin{abstract}
RESUMO
Os métodos mecanísticos de projeto de pavimentos admitem perfeita aderência entre as camadas, o que pode não ocorrer no campo. Neste trabalho, com uso do programa de elementos finitos ABAQUS, são analisados os efeitos da condição de interface das camadas (aderência completa, parcial e nula) no comportamento mecânico de pavimentos. As análises foram realizadas para diferentes níveis de rigidez do material asfáltico e a estimativa do desempenho de pavimentos flexíveis, tanto a fadiga das camadas asfálticas quanto a deformação permanente, foi baseada em equações empírico-mecanísticos que consideram, respectivamente, as respostas estruturais: deformação horizontal de tração na fibra inferior das camadas asfálticas e deformação vertical de compressão no topo do subleito. Os resultados mostram que as respostas estruturais são mais sensíveis à condição de aderência na interface entre as camadas asfálticas, para a qual contribui a pintura de ligação, do que na segunda interface. A condição de interface entre as camadas afeta mais a vida do pavimento à fadiga que à deformação permanente $e$ em ambos os casos a consideração de perfeita aderência entre as camadas resulta na previsão de melhor desempenho dos pavimentos. Portanto, o surgimento prematuro de defeitos pode estar relacionado, também, à fraca aderência entre camadas.
\end{abstract}

\section{ABSTRACT}

The mechanistic analysis for pavement design admit perfect adherence between the layers, which may not occur in the field. In this work, using the ABAQUS finite element program, the effects of the layer interface condition (complete, partial and zero adhesion) on the behavior of pavements are analyzed. The analyzes were carried out for different levels of stiffness of the asphalt material and the estimation of the performance of flexible pavements, in terms of fatigue of the asphaltic and cemented layers and permanent deformation, was based on empiric-mechanistic equations that consider, respectively, the structural responses: horizontal tensile strain at the bottom fiber of the asphalt layers and vertical compressive strain at the top of the subgrade. The results show that the structural responses are more sensitive to the adhesion condition at the interface between of the two asphalt layers, to which the tack coat contribute the most, than at the second interface. The interface condition affects the fatigue life of the pavement more than the permanent deformation and, in both cases, the consideration of perfect adhesion between the layers results in the prediction of better pavement performance. Therefore, the premature occurrence of distresses may also be related to poor adherence between layers.

\section{INTRODUÇÃO}

Os métodos mecanísticos de projeto para pavimentos flexíveis são baseados nas tensões, deformações e deslocamentos que ocorrem no pavimento quando sujeitos ao carregamento do tráfego. Para simplificar o cálculo desses parâmetros, é comum considerar que as camadas estão perfeitamente conectadas entre si, o que permite analisar o pavimento como uma estrutura monolítica. 
Em campo, para se aproximar dessa condição, é comum se utilizar uma pintura de ligação, cuja eficiência depende de diversos fatores como as propriedades do material asfáltico empregado e sua taxa de aplicação (Raab et al., 2016). Mesmo com o auxílio dessas técnicas, uma conexão adequada nem sempre é alcançada e problemas relacionados à falha de aderência entre as camadas têm sido reportados em diversos estudos (Raab e Partl, 2004 e Sutanto, 2010).

Trincas por deslizamento ou deformação horizontal na superfície do pavimento são as evidências mais comuns de defeitos associados à falha de aderência entre as camadas. Problemas de deformação permanente horizontal foram observados por Sutanto, em 2001 na Indonésia, em alguns locais de frenagem e conversão dos veículos (Sutanto, 2010). Raab e Partl (2004) relataram casos de trincas por deslizamento entre as camadas e deformação permanente horizontal relacionados à uma pobre condição de contato na interface inferior do revestimento.

Trincas por deslizamento das camadas também foram observadas em um projeto de recapeamento em Nevada, nos Estados Unidos. 0 defeito alcançou aproximadamente $40 \%$ da extensão do projeto pouco tempo depois da conclusão da obra (Charmot, 2005).

Os danos típicos do pavimento causados por uma fraca ligação entre as camadas asfálticas ocorrem, comumente, em áreas de frenagem e aceleração devido aos esforços horizontais atuantes na estrutura. Contudo, os efeitos da falha de aderência no pavimento também são notados quando se admite, somente, esforços verticais (Romanoschi e Metcalf, 2001a).

A falha de aderência entre camadas, mesmo sob a consideração apenas de cargas verticais, provoca uma alteração no estado de tensões e deformações da estrutura. King e May (2004) apresentaram uma análise dos efeitos da adesão entre camadas de misturas asfálticas, com o uso do software BISAR, sob duas intensidades diferentes de carregamentos e verificaram um aumento significativo no quadro de tensões do pavimento.

Problemas de aderência em pavimentos não ocorrem apenas entre a camada de revestimento e a camada de binder (Raab e Partl, 2004). Ziari e Kabiri (2007) também chegaram a essa conclusão quando, com o software KENLAYER, modelaram uma estrutura de pavimento composta por uma camada asfáltica superficial, camada asfáltica intermediária (binder) e uma base granular. Os autores verificaram um aumento nas tensões verticais de compressão no topo da camada de subleito quando consideram a não aderência entre as camadas.

Kruntcheva et al. (2005) desenvolveram um estudo teórico sobre os efeitos da condição de interface no comportamento mecânico de um pavimento com estrutura semelhante à estudada por Ziari e Khabiri (2007). Os autores consideraram, além das condições extremas de aderência, uma condição parcial de contato entre as camadas. Por meio do programa BISAR, a condição parcial de aderência foi definida pelo módulo de reação ao cisalhamento horizontal (K). No caso mais extremo, a vida do pavimento com camadas não aderidas pode ser de até $80 \%$ da vida do pavimento em que as camadas estão completamente aderidas.

Wu et al. (2017) modelaram três condições de contato na interface (aderência total, parcial e nula) e analisaram a deflexão, a tensão de tração por flexão na fibra inferior da camada asfáltica e a tensão de cisalhamento no topo da camada de base. Foi verificado que perda da aderência entre as camadas chegou a alterar o estado de tensões da camada asfáltica de compressão para tração nos casos mais críticos.

A avaliação dos efeitos da condição de interface na resposta do pavimento tem sido verificada por meio de modelos mecanísticos com o amparo de ferramentas computacionais. Mesmo com o avanço dessas ferramentas, poucos dentre os softwares mais utilizados na análise de pavimentos possibilitam a modelagem do contato entre as camadas. Diante disso, diversos estudos têm 
recorrido à utilização de ferramentas com uma proposta de análise mais ampla, como o programa ABAQUS, um dos programas de uso geral mais utilizado para resolver problemas de engenharia, como análise de tensões de deformações e problemas de transferência de calor com base no Método dos Elementos Finitos.

A aplicação dessa ferramenta ao estudo de pavimentos tem sido intensa nos últimos anos. Cho (1996) apontou algumas considerações da modelagem de pavimento no ABAQUS em seu estudo. Três tipos de modelos foram abordados: Tridimensional (3-D); Bidimensional (2-D) e Axissimétrico. $\mathrm{O}$ autor indica este último como uma boa escolha para o estudo de pavimentos com a aplicação de um eixo simples e roda simples, porém ressalta que o modelo tridimensional é a melhor opção ao se aplicar um eixo simples de rodas duplas. Essas conclusões também foram tiradas por Yassenn et al. (2015) em um estudo semelhante.

Uddim et al. (1994), com a utilização do ABAQUS, investigaram a resposta de pavimentos com trincas sob carregamento dinâmico e compararam os resultados com as repostas de um sistema elástico linear sob carregamento estático. Sukumaran (2004) apresentou uma análise tridimensional de pavimentos aeroportuários com o uso do ABAQUS onde abordou a não linearidade dos materiais. A condição de não linearidade dos materiais que compõem as camadas de um pavimento também foi modelada com a mesma ferramenta por Kim (2009). Wu et al. (2011) também fez uso do software para modelar um modelo de previsão de deformação permanente em pavimento com base e sub-base estabilizadas com materiais cimentantes.

Os diversos estudos realizados com o ABAQUS demonstram a ampla utilização do software na pavimentação e expõem os benefícios de se utilizar um programa de proposta geral, como a consideração da descontinuidade das camadas e a modelagem da interface de contato entre elas (Romanoshi e Metcalf, 2001b).

Com base nisso, o trabalho busca verificar a influência da interação entre as camadas do pavimento em seu comportamento estrutural, ao aplicar o ABAQUS em uma análise linear de um pavimento flexível sob condições de aderência completa, parcial e nula.

\section{MÉTODO}

Os pavimentos abordados aqui foram modelados com elementos finitos, por meio do software ABAQUS, o qual permitiu estimar o comportamento mecânico das estruturas sob diferentes condições de aderência entre as camadas asfálticas. Foi feita uma análise linear em que se admitiu estruturas com materiais homogêneos e isotrópicos, o que faz do módulo de resiliência e o coeficiente de Poisson os principais parâmetros que descrevem o comportamento mecânico desses materiais.

\subsection{Modelagem tridimensional em Elementos Finitos}

O ABAQUS é um conjunto de instrumentos de análises de proposta geral que permite avaliar uma grande variedade de problemas da engenharia. A modelagem no ABAQUS exige do usuário um número maior de dados em seus ficheiros de entrada, por se tratar de um programa genérico, porém o software conta com uma interface que possibilita a modelagem de forma fácil e rápida, além de uma exibição gráfica da distribuição de tensões e deformações do elemento analisado, como pode ser visto na Figura 1.

A modelagem da geometria do problema baseia-se em um sistema cartesiano de referência no espaço, em que o eixo X corresponde à direção de tráfego da rodovia, o eixo Y à direção transversal da rodovia e o eixo $\mathrm{Z}$ normal à superfície de rolamento. Devido aos custos computacionais 
atrelados às simulações, foi levado em conta a simetria em X do problema analisado, o que implicou na modelagem apenas do semieixo. 0 subleito foi admitido com uma espessura de $4 \mathrm{~m}$, como é adotado no programa FEPAVE2 (Medina e Motta, 2015), e as dimensões do pavimento no plano XY foram fixadas em $2 \mathrm{~m}$ de largura e $2 \mathrm{~m}$ de comprimento.

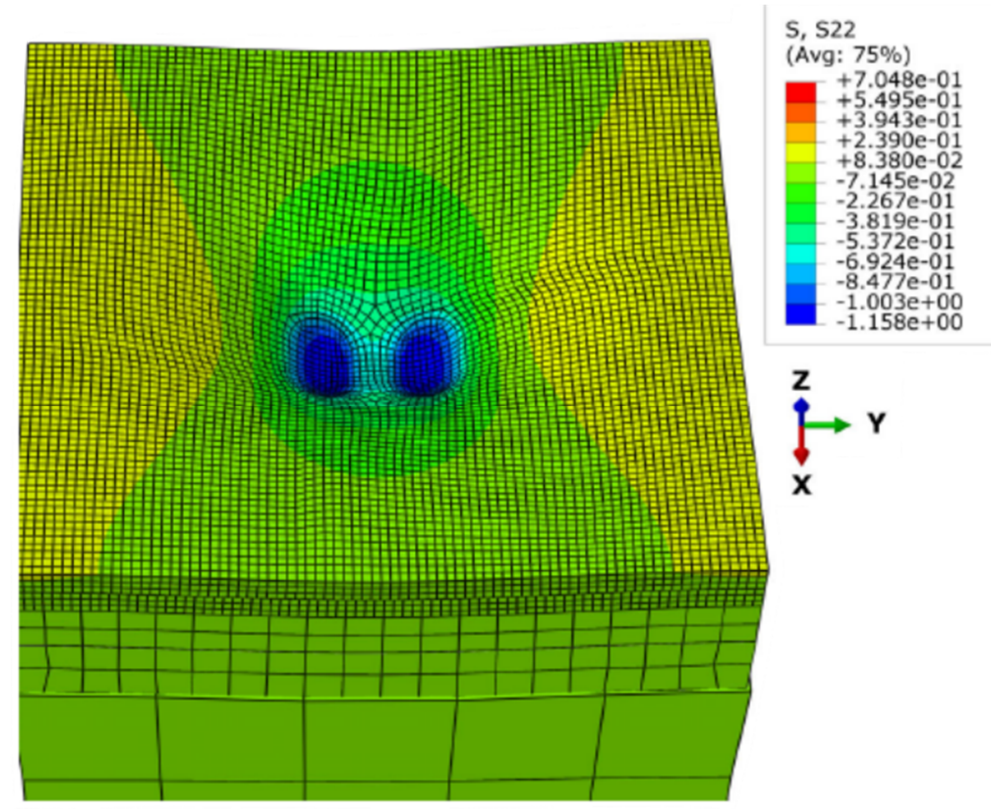

Figura 1: Distribuição das tensões horizontais de tração - ABAQUS

O ABAQUS oferece algumas ferramentas que permitem determinar as condições de aderência entre duas superfícies citadas anteriormente. Por padrão, para modelar o contato entre duas superfícies, o software usa um algoritmo de contato em que é necessário a classificação das superfícies em slave e master. No algoritmo, os nós em uma superfície (slave surface) não conseguem penetrar os segmentos que compõem a outra superfície (master surface).

A condição de aderência total entre as camadas foi modelada com a opção "tie constraint" para tornar o par de camadas subsequentes uma estrutura monolítica. Nessa opção de contato, cada nó na superfície slave é restrito ao mesmo movimento que o nó mais próximo na superfície master. Para uma análise estrutural, isso significa a restrição dos graus de liberdade de translação e rotação.

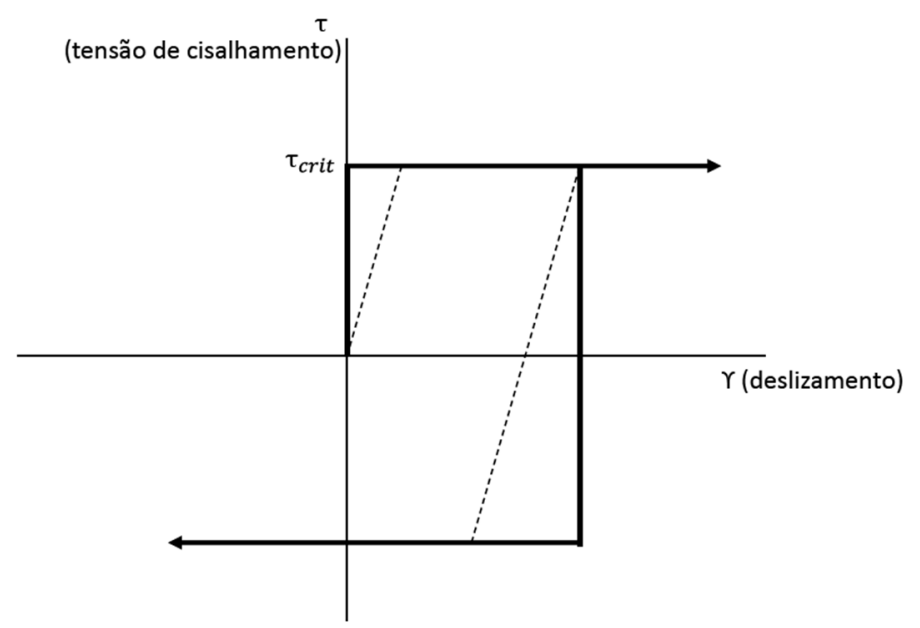

Figura 2: Comportamento da interface pelo modelo de atrito de Coulomb. (ABAQUS, 2014) 
O modelo de Coulomb foi utilizado apara descrever o comportamento da interface nas condições de aderência parcial e nula entre as camadas. 0 conceito básico desse modelo é relacionar a tensão de cisalhamento máxima permitida na interface com a pressão de contato entre as camadas. Na forma básica do modelo de Coulomb, duas superfícies em contato podem transmtir tensões de cisalhamento até uma certa magnitude $\left(\tau_{\text {crit }}\right)$ antes de começarem a se deslocar entre si, como pode ser visto na Figura 2, onde as linhas sólida e pontilhada mostram o comportamento da interface antes e depois que a tensão cisalhante atinge a tensão crítica, respectivamente.

O modelo de Coulomb define a tensão crítica de cisalhamento como uma fração da pressão de contato entre as superfícies ( $p$ ), como representado na Equação 1.

$$
\tau_{\text {crit }}=\mu \mathrm{p}
$$

em que o $\mu$ é o coeficiente de atrito, normalmente associados a valores entre 0 e 1 , e considerado o mesmo em todas as direções (coeficiente isotrópico). A condição parcial de contato entre as camadas foi modelada com o coeficiente de atrito igual a 0,5. Para representar o caso de camadas não aderidas, reduziu-se o valor de $\mu$ a zero, o que permite o livre deslocamento relativo entre as camadas.

Apesar de não considerar o efeito de colagem promovido pelo material asfáltico, o modelo de Coulomb possibilita, de maneira simplificada, a estimativa da variação da resistência ao deslocamento relativo entre as camadas, permitindo a inferência dos efeitos que diferentes condições de aderência entre as camadas têm sobre o comportamento estrutural dos pavimentos.

O ABAQUS, ao utilizar o Método dos Elemento Finitos, subdivide o meio contínuo em elementos finitos delimitados pelos nós, onde são calculadas as tensões, deformações e deslocamentos ao longo da estrutura. Em função disso, a condição de deslizamento, definida pela Equação 1, também é verificada em cada ponto de análise (em cada nó), podendo haver nós com diferentes deslocamentos relativos.

Três condições de contorno foram aplicadas na modelagem das estruturas de pavimentos. 0 fundo do subleito foi engastado, ou seja, impediu-se qualquer movimentação rotacional e de translação em todas as direções. As faces laterais no plano XZ das camadas foram impedidas de se movimentar no eixo $\mathrm{Y}$ e as faces laterais no plano YZ, no eixo X (Figura 3).
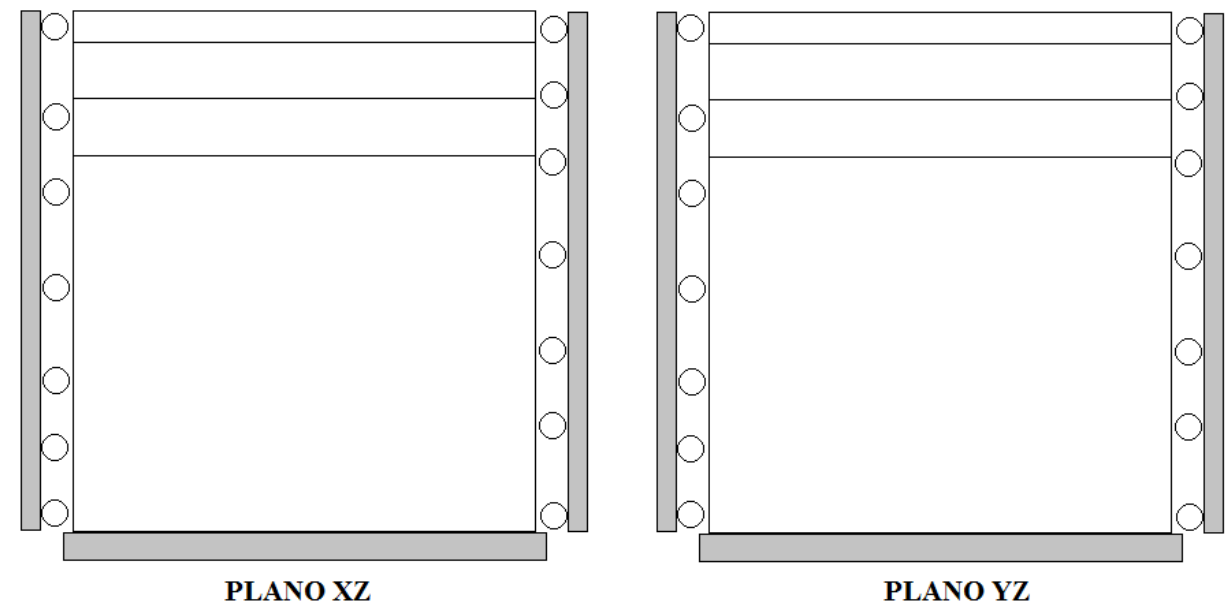

Figura 3: Condições de contorno 
Devido ao tempo de simulação e memória requerida na modelagem das estruturas dos pavimentos, utilizou-se uma malha não homogênea, com sua maior densidade de elementos concentrada nas camadas asfálticas. A malha foi composta por elementos denominados C3D8R. Essa nomenclatura representa elementos sólidos contínuos que possuem 8 nós e têm os dados de coordenadas fora dos nós obtidos por meio de uma interpolação linear.

Por meio dos resultados obtidos nas simulações computacionais, se avaliou as tensões, deformações e deslocamentos mais representativos quanto aos critérios de fadiga e deformação plástica do subleito, ou seja, a deformação por tração na face inferior do revestimento e a deformação vertical por compressão no topo do subleito.

\subsection{Caracterização da estrutura do pavimento}

A estrutura analisada nesse trabalho consiste em um pavimento com duas camadas asfálticas iguais, sobrepostas às camadas de base, sub-base e subleito. As camadas asfálticas são compostas por concreto asfáltico. Para a camada de base, foi admitido uma brita graduada simples (BGS) e macadame seco e solo arenoso para as camadas de sub-base e subleito, respectivamente. A geometria e as propriedades dos materiais das estruturas analisadas são mostradas na Tabela 1.

Tabela 1: Propriedades geométricas e de materiais da estrutura do pavimento

\begin{tabular}{lllll}
\hline Camada & Material & Espessura $(\mathbf{c m})$ & MR (MPa) & v \\
\hline 1 & Concreto asfáltico & 7,5 & $3000 / 7000 / 11000$ & 0,3 \\
2 & Concreto asfáltico & 7,5 & $3000 / 7000 / 11000$ & 0,3 \\
3 & BGS & 15 & 300 & 0,4 \\
4 & M. Seco & 20 & 200 & 0,4 \\
5 & Solo arenoso & $\infty$ & 100 & 0,45 \\
\hline
\end{tabular}

As interfaces entre as duas camadas asfálticas e entre a camada asfáltica e a base do pavimento foram modeladas sob três condições de aderência diferentes:

- Aderência completa (sem deslocamento relativo - $\mu=1$ )

- Aderência parcial $(\mu=0,5)$

- Aderência nula $(\mu=0)$

Para avaliar a sensibilidade da resposta estrutural dos pavimentos às condições de interação entre as camadas, as condições de aderência foram combinadas em nove casos diferentes, apresentados na Tabela 2. As interfaces não contidas na Tabela 2 foram admitidas com aderência total entre as partes (condição 1 de aderência).

Tabela 2: Condições de aderência na interface

\begin{tabular}{lll}
\hline \multirow{2}{*}{ Casos } & Revestimento & CA inferior \\
\cline { 2 - 3 } & CA inferior & Base \\
\hline A & 1 & 1 \\
B & 0,5 & 1 \\
C & 0 & 1 \\
D & 1 & 0,5 \\
E & 0,5 & 0,5 \\
F & 0 & 0,5 \\
G & 1 & 0 \\
H & 0,5 & 0 \\
I & 0 & 0 \\
\hline
\end{tabular}




\subsection{Condições de carregamento}

As cargas do tráfego foram modeladas com a configuração geométrica de um eixo simples de rodas duplas (Figura 4). A magnitude da carga de cada roda e da pressão de contato pneu-pavimento foi de $48,5 \mathrm{kN}$ e $720 \mathrm{kPa}$, respectivamente. A pressão de contato entre o pneu e o pavimento foi considerada uniforme e o carregamento implementado levou em consideração unicamente as componentes verticais das cargas do tráfego.

A geometria da superfície de contato entre o pneu e o pavimento seguiu a recomendação de Huang (2004). 0 autor aponta uma elipse, modelada como a união entre um retângulo e duas semicircunferências, como a melhor forma geométrica de representar a área de aplicação das cargas do tráfego (Figura 4).

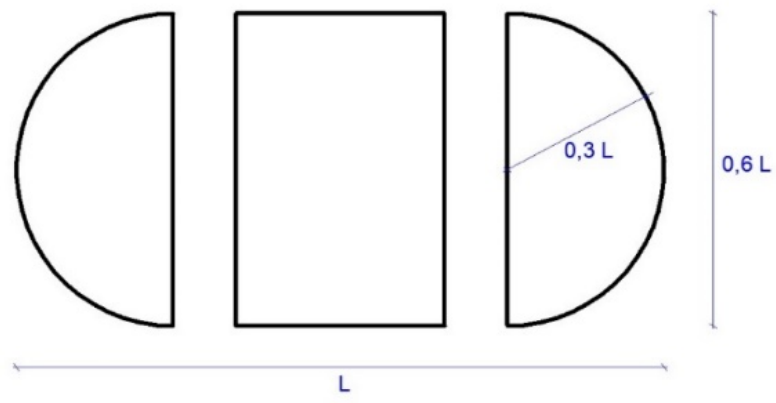

Figura 4: Área de contato pneu-pavimento

\subsection{Comportamento dos pavimentos asfálticos}

Por meio dos resultados obtidos nas simulações computacionais (tensões, deformações e deslocamentos), foi avaliada a influência da condição de interface entre camadas no comportamento do pavimento para cada caso apresentado na Tabela 2. Também foi verificado o efeito da condição de interface na vida do pavimento à fadiga e à deformação permanente por meio dos modelos de previsão de desempenho.

Os números de solicitações admissíveis à fadiga $\left(N_{f a d}\right)$ e à deformação permanente $\left(N_{\text {def }}\right)$ foram calculados com modelos de previsão de desempenho dependentes nas respostas estruturais do pavimento. Os desempenhos do pavimento à fadiga e à deformação permanente foram estimados com as equações apresentadas no método da Shell International Petroleum (SHELL, 1998), Equações 2 e 3 respectivamente.

$$
\begin{gathered}
N_{f a d}=0,0685 M R^{-2,363}\left(\varepsilon_{h}\right)^{-5,671} \\
N_{d e f}=\left(\frac{0,028}{\varepsilon_{v}}\right)^{4}
\end{gathered}
$$

em que $\varepsilon_{h}$ representa a deformação horizontal de tração máxima na fibra inferior da camada asfáltica e $\varepsilon_{V}$ é a deformação vertical de compressão máxima no topo do subleito.

\section{ANÁLISE DOS RESULTADOS}

Os resultados obtidos com as simulações computacionais foram submetidos a um experimento fatorial em que se assumiu três fatores (módulo de rigidez do material asfáltico -MR, aderência na interface entre camadas asfálticas - i1 e aderência entre a camada asfáltica e a base - i2). As 
variáveis independentes supracitadas, apesar de naturalmente serem variáveis contínuas, foram abortadas como variáveis discretas separadas em três classes cada uma delas. As variáveis dependentes são representadas pelas respostas estruturais do pavimento e foram analisadas separadamente, por estarem associados a diferentes mecanismos de deterioração estrutural.

\subsection{Análise de regressão}

No experimento fatorial, foi realizada uma análise de regressão para tentar relacionar quantitativamente as variáveis independentes (Fatores MR, $\mathrm{i}_{1} \mathrm{e} \mathrm{i}_{2}$ ) com as respostas estruturais do pavimento, através dos desenvolvimentos de modelos de regressão linear múltipla. As equações de regressão das respostas estruturais do revestimento, da camada asfáltica inferior e do subleito são apresentadas nas Equações 4 a 6, respectivamente, e os coeficientes de determinação desses modelos obtidos estão dispostos na Tabela 3. A partir dos resultados dos coeficientes de determinação, observou-se que os erros dos modelos de regressão associados aos fatores MR, i1 e i2 são inferiores a $12 \%$.

$$
\begin{aligned}
& \varepsilon_{h, C A_{-} 1}\left(E, i_{1}, i_{2}\right)=135,12+38,3 M R(3)+1,5 M R(7)-39,8 M R(11)+101,7 i_{1}(0)+40,9 i_{1}(0,5)-142,6 i_{1}(1) \\
& +18,7 i_{2}(0)-2,1 i_{2}(0,5)-16,6 i_{2}(1) \\
& \varepsilon_{h, C A_{-} 2}\left(E, i_{1}, i_{2}\right)=191,97+70,21 M R(3)+15,21 M R(7)-55 M R(11)+2,41 i_{1}(0)-15,96 i_{1}(0,5)+13,54 i_{1}(1) \\
& +30,39 i_{2}(0)+7,33 i_{2}(0,5)-37,72 i_{2}(1) \\
& \varepsilon_{v, \text { subleit }}\left(E, i_{1}, i_{2}\right)=-28514-33,53 M R(3)+4,32 M R(7)+29,21 M R(1)+34,8 i_{0}(0)-23,2 i_{1}(0,5)+58,1 i_{1}(1) \\
& -27,3 i_{2}(0)-13,9 i_{2}(0,5)+41,3 i_{2}(1)
\end{aligned}
$$

Tabela 3: Coeficientes de determinação dos modelos de regressão

\begin{tabular}{ll}
\hline Modelo & $\mathbf{R}^{\mathbf{2}}$ \\
\hline$\varepsilon_{\mathrm{h}, \mathrm{CA}_{-} 1}$ & 91,89 \\
$\varepsilon_{\mathrm{h}, \mathrm{CA} \_2}$ & 88,77 \\
$\varepsilon_{\mathrm{v}, \text { subleito }}$ & 97,29 \\
\hline
\end{tabular}

\subsection{Análise de significância}

Neste trabalho, o nível de significância dos fatores foi determinado por meio do P-valor, associado a um nível de confiança de 95\%. Os valores calculados para cada resposta estrutural estão dispostos na Tabela 4.

Tabela 4: P-Valor

\begin{tabular}{llll}
\hline Modelo & \multicolumn{3}{l}{ Base de BGS } \\
\cline { 2 - 4 } & $\mathbf{E}$ & $\mathbf{i}_{1}$ & $\mathbf{i}_{2}$ \\
\hline$\varepsilon_{\mathrm{h}, \mathrm{CA} \_1}$ & 0,001 & 0 & 0,165 \\
$\varepsilon_{\mathrm{h}, \mathrm{CA} \_2}$ & 0 & 0,063 & 0 \\
$\varepsilon_{\mathrm{v}, \text { subleito }}$ & 0 & 0 & 0 \\
\hline
\end{tabular}

Com base nas tabelas apresentadas, é possível verificar que, na maioria dos casos, uma parcela das alterações das respostas estruturais calculadas pode ser explicada pela alteração na 
condição de aderência entre as camadas do pavimento, ou seja, a condição de aderência entre as camadas altera as respostas estruturais do pavimento. Dessa forma, no caso de construção da camada de um pavimento, deve-se ater à integridade da espessura projetada, uma vez que a substituição de uma camada única por duas camadas de espessura total equivalente não representa a mesma condição de esforços internos, tendo em vista que a perfeita aderência entre as camadas não é alcançada em campo.

\subsection{Comparação entre as condições de interface}

As deformações máximas foram estimadas por meio dos modelos de regressão e os resultados encontrados são apresentados em forma de gráfico (Figura 5), para melhor compreensão e praticidade em identificar os efeitos da condição de contato entre as camadas no comportamento estrutural dos pavimentos.

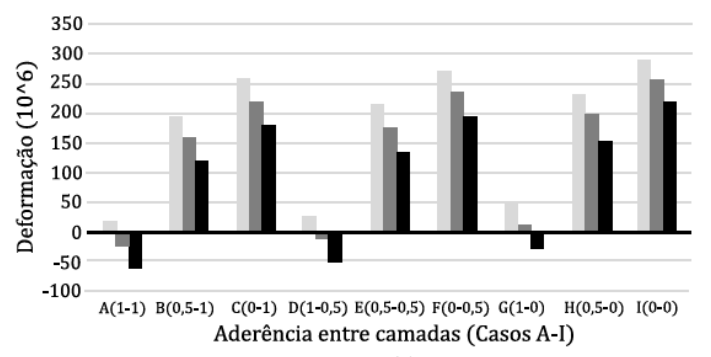

(a)

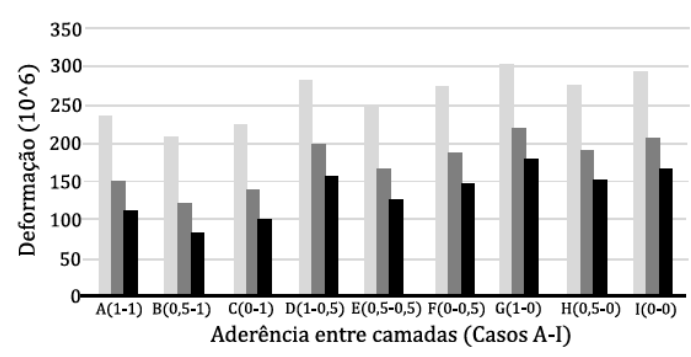

(b)

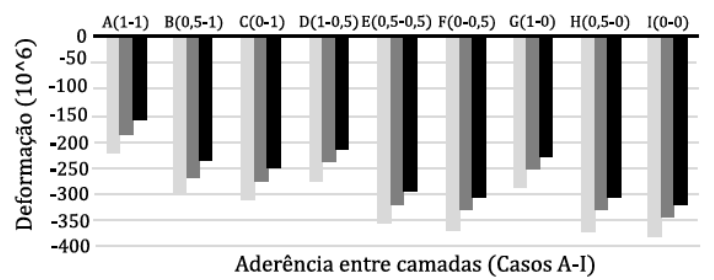

(c)

Figura 5: Deformações máximas: (a) camada de revestimento, (b) camada asfáltica inferior e (c) subleito

A deformação horizontal no revestimento (Figura 5 (a)), em alguns casos, se apresentou no estado de compressão quando ambas as interfaces estavam perfeitamente aderidas (Caso A). A perda de aderência nas duas interfaces (Caso I), por sua vez, resultou em maiores deformações e levou à deformação horizontal no revestimento ao estado de tração. As deformações máximas ocorridas na camada de revestimento foram mais sensíveis à ligação entre as camadas asfálticas que entre a camada asfáltica e a base do pavimento.

A deformação vertical de compressão no topo do subleito (Figura 5 (c)) foi mais sensível à condição da interface entre as camadas asfálticas que entre a camada asfáltica e a base do pavimento. A perda total de aderência acarretou em deformação vertical de compressão aproximadamente duas vezes maior que a deformação encontrada no Caso A (aderência completas nas duas interfaces).

A camada de revestimento e o subleito apresentaram as menores deformações quando havia uma perfeita aderência em ambas as interfaces. No entanto, isso não foi notado na camada asfáltica inferior (Figura 5 (c)), o que pode ter ocorrido em função da alteração na distribuição de tensões ao longo da espessura da camada, provocada pela variação da condição de interface. 
As curvas na Figura 6 mostram que a perda de aderência levou a tensão horizontal, que tendia ao estado de tração ao longo da espessura, a voltar ao estado de compressão no topo da camada inferior, o que resultou em menores tensões máximas na fibra inferior dessa camada.

A perda de aderência entre as camadas resultou na variação da localização da deformação horizontal crítica, ou seja, a camada que atingirá a condição crítica à fadiga variou entre o revestimento e a camada asfáltica inferior em função do nível de aderência entre as camadas.

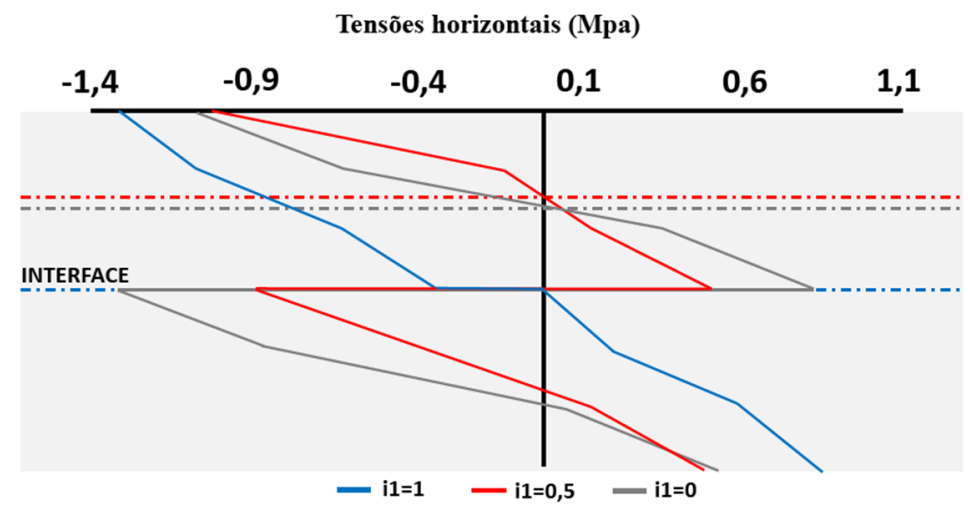

Figura 6: Distribuição de tensões ao longo da espessura das camadas asfálticas

\subsection{Previsão de desempenho}

As respostas estruturais calculadas com os modelos de regressão foram associadas ao número de solicitações admissíveis à fadiga e à deformação permanente, por meio dos modelos de previsão de desempenho. Os valores de deformação apresentados no item anterior indicam que quando a condição de perfeita aderência entre as camadas não é alcançada, a distribuição de tensões e deformações muda expressivamente, o que afeta também a previsão de desempenho do pavimento como disposto nas Figuras 7 e 8.

0 número de solicitações responsável por levar o pavimento à ruptura por fadiga é apresentado na Figura 7. Para pavimentos com material asfáltico mais rígido (7 e $11 \mathrm{mil} \mathrm{MPa}$ ), o caso de completa aderência entre as camadas (Caso A) resultou em uma vida de fadiga maior que os demais casos e alcançou um valor aproximadamente 170 vezes maior que o caso em que as camadas não estavam aderidas (Caso I).

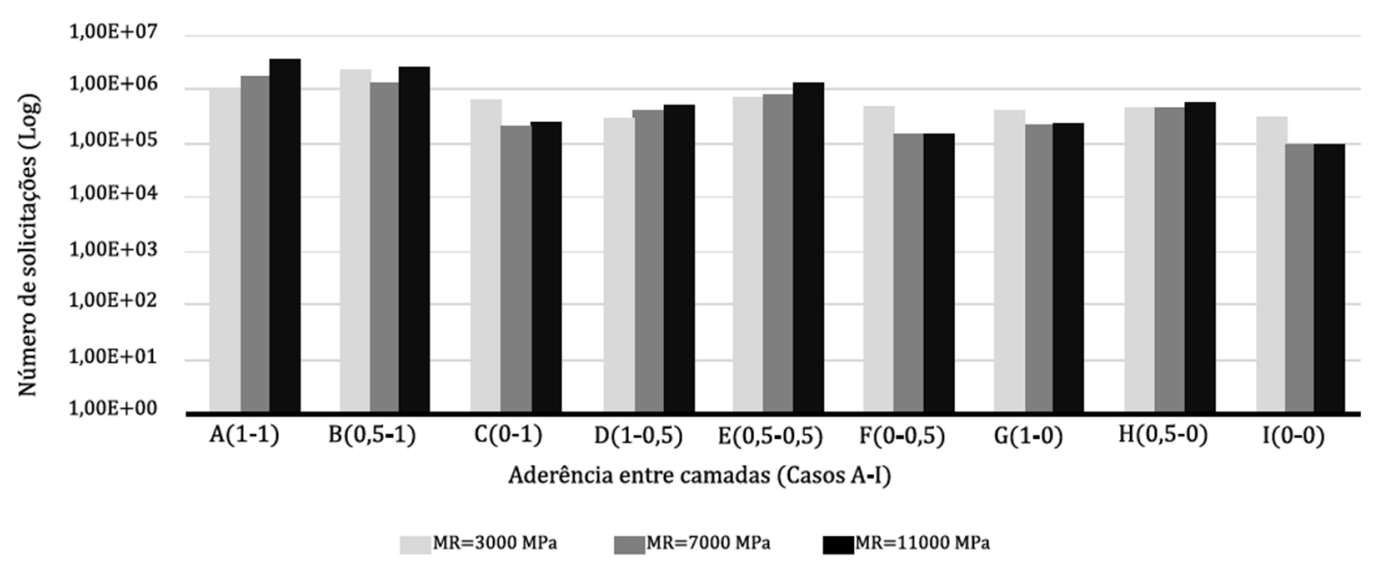

Figura 7: Solicitações admissíveis à fadiga 
No entanto, para materiais com menor rigidez, observou-se que a perda parcial de aderência entre as camadas asfálticas resultou em uma maior vida e fadiga, devido às menores deformações máximas sofridas, o que aponta a necessidade de maiores investigações a respeito da relação entre a rigidez do material, principalmente com baixa rigidez, e o comportamento mecânico da interface, por meio de estudos experimentais. No quadro geral, pode-se verificar que vida de fadiga do pavimento foi afetada de forma semelhante pelas duas interfaces estudadas.

Quanto à deformação permanente (Figura 8), a vida do pavimento também foi maior quando as camadas estavam completamente aderidas (Caso A) sendo aproximadamente 35 vezes maior que o caso I (camadas não aderidas). Dentre as duas interfaces analisadas, o número de solicitações admissíveis à deformação permanente foi mais sensível à condição de contato entre a camadas asfáltica inferior e a base do pavimento.

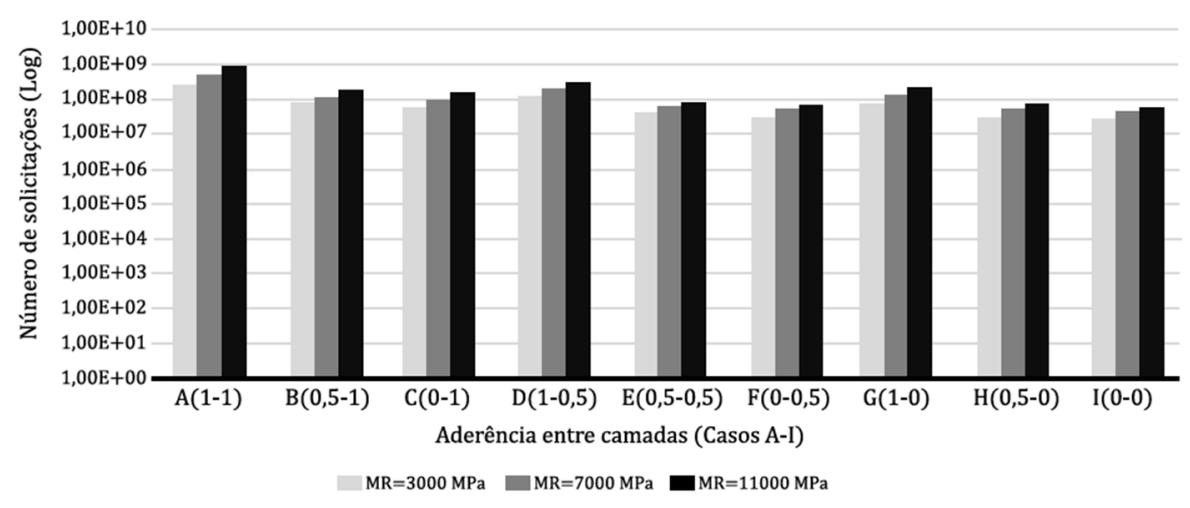

Figura 8: Solicitações admissíveis à deformação permanente", respectivamente.

Na Tabela 5, é possível verificar a redução máxima (em \%) do número de solicitações admissíveis, atribuída a variação de condição de aderência, para cada nível de rigidez do material asfáltico. $\mathrm{O}$ efeito da interação entre camadas na vida do pavimento à fadiga e à deformação permanente esteve diretamente relacionada à rigidez do material, de maneira que quanto mais rígido o material asfáltico, maior foi a influência da aderência entre camadas na capacidade do pavimento em resistir as solicitações do tráfego.

Tabela 5: Redução máxima do número $\mathrm{N}$

\begin{tabular}{lll}
\hline & MR(MPa) & Redução Máx. \\
\hline \multirow{3}{*}{ Nfad } & 3000 & $88,7 \%$ \\
& 7000 & $94,8 \%$ \\
& 11000 & $97,5 \%$ \\
\hline \multirow{3}{*}{ Ndef } & 3000 & $89,0 \%$ \\
& 7000 & $92,2 \%$ \\
& 11000 & $94,1 \%$ \\
\hline
\end{tabular}

\section{CONCLUSÕES}

Este trabalho teve como objetivo verificar o comportamento mecânico de estruturas de pavimentos flexíveis sob diferentes condições de interface entre camadas e sua influência sobre o desempenho previsto a partir de respostas estruturais associadas ao aparecimento e propaga- 
ção de trincas por fadiga e ao acúmulo de deformação permanente nas trilhas de roda controlada pela deformação vertical do subleito.

A condição de aderência nas duas primeiras interfaces das camadas do pavimento, principalmente na interface das camadas asfálticas, alterou de forma significativa as respostas estruturais do revestimento e do subleito do pavimento. Consequentemente, a falta de aderência entre essas camadas resulta no comprometimento do desempenho dos pavimentos à fadiga e à deformação permanente.

0 efeito da condição de interface entre as camadas altera as deformações horizontais máximas não só em magnitude, como também na sua localização ao longo da estrutura, ou seja, a condição crítica à fadiga do pavimento pode ser alcançada por camadas diferentes em decorrência da condição de aderência na interface entre as camadas.

Ficou evidente o impacto da condição de interface entre as camadas na vida do pavimento e que há influência da rigidez do material das camadas. Quanto mais rígido o material asfáltico, maior é o efeito da condição de aderência entre as camadas na vida do pavimento à fadiga e à deformação permanente.

A consideração de perfeita aderência entre as camadas resulta na previsão de melhor desempenho dos pavimentos. Portanto, o surgimento prematuro de defeitos pode estar relacionado, também, à fraca aderência entre camadas, ressaltando a importância de todos os recursos que colaboram para melhorar a condição de contato na interface entre camadas, como, por exemplo, a pintura de ligação.

Os resultados obtidos neste trabalho evidenciam a importância da condição de interface entre as camadas de um pavimento asfáltico, pois afetam, significativamente, as respostas estruturais e devem, portanto, ser adequadamente consideradas nos métodos mecanísticos de dimensionamento de pavimentos, ou seja, de acordo com as condições que efetivamente ocorrerão nos pavimentos em serviço.

As equações de previsão de desempenho dos pavimentos abordadas aqui, em sua concepção, não consideraram as condições de aderência entre as camadas e, consequentemente, seus coeficientes podem ser sensíveis à condição de interface, o que pode distanciar os modelos de uma boa representação para a estimativa da vida do pavimento em virtude das reais condições de aderência verificadas em campo. Em razão disso, associado aos resultados encontrados no trabalho, ressalta-se a importância da incorporação da condição de interface nas análises dos pavimentos em serviço dos estudos em desenvolvimento para a elaboração de novos modelos de previsão de desempenho.

\section{REFERÊNCIAS}

ABAQUS (2014) Getting Started with ABAQUS, Keywords Edtion 14, 2014. Disponível em http://abaqus.software.polimi.it/v6.14/books/gsk/default.htm (acesso em 22/08/2019)

Charmot S.; P. Romero e M. Dunning (2005) Forensic analysis of slippage cracking. Anais do $84^{\circ}{ }^{\circ}$ Transportation Research Board. Washington DC, USA.

Cho, Y. H.; B. McCullough e J. Weissmann (1996) Considerations on Finite-Element Method Application in Pavement Structural Analysis. Transportation Research Record: Journal of the Transportation Research Board, 1539, 96-101. doi:10.3141/153913.

Wu, Z.; X. Chen; X. Yang e Z. Zhang (2011) Finite Element Model for Rutting Prediction of Flexible Pavement with Cementitiously Stabilized Base-Subbase. Transportation Research Record: Journal of the Transportation Research Board, 2226(1), 104-110. doi:10.3141/2226-11

Huang, Y. H. (2004) Pavement Analysis and Design, 2ํe. Pearson Prentice Hall, New Jersey, USA.

Kim, M.; E. Tutumluer e J. Kwon (2009) Nonlinear Pavement Foundation Modeling for Three-Dimensional Finite-Element Analysis of Flexible Pavements. International Journal of Geomechanics, 9(5), 195-208. doi:10.1061/(asce)15323641(2009)9:5(195) 
King, G. e R. May (2004) New Approaches to Tack Application, 83ํㅡㄹ Annul Meeting of the Transportation Research Board, Washington, D.C.

Kruntcheva, M. R.; A. C. Collop e N. H. Thom (2005) Effect of Bond Condition on Flexible Pavement Performance. Journal of Transportation Engineering, 131(11), 880-888. doi:10.1061/(asce)0733-947x(2005)131:11(880)

Medina, J. e L.M.G. Motta (2015) Mecânica dos pavimentos. Ed. Interciência, Rio de Janeiro.

Raab C e M. N. Partl (2004) Interlayer shear performance: experience with different pavement structures. Anais da $3^{\circ} \stackrel{\text {. Euras- }}{ }$ phalt \& Eurobitume Congress, Vienna, Austria, p.535-545. Disponível em < https://trid.trb.org/view/743792> (acesso em 22/08/2019)

Raab, C.; J. Grenfell e A. O. Abd El Halim e M.N. Partl (2016) Comparison of Interlayer Bond Behavior Due to Ageing. $8^{o}$ International RILEM SIB Symposium, Ancona, p. 7-9. doi:10.1007/978-94-017-7342-3_26

Romanoschi, S. e J. Metcalf (2001a) Effects of Interface Condition and Horizontal Wheel Loads on the Life of Flexible Pavement Structures. Transportation Research Record: Journal of the Transportation Research Board, 1778, 123131. doi:10.3141/1778-15

Romanoschi, S. e J. Metcalf (2001b) Characterization of Asphalt Concrete Layer Interfaces. Transportation Research Record: Journal of the Transportation Research Board, 1778, 132-139. doi:10.3141/1778-16

SHELL (1998) Bitumen Stress Analysis in Roads BISAR 3.0 User's Manual. Shell International

Petroleum Company Limited.

Sukumaran, B.; M. Willis e N. Chamala (2005) Three Dimensional Finite Element Modeling of Flexible Pavements. Advances in Pavement Engineering. doi:10.1061/40776(155)7

Sutanto M. (2010) Assessment of bond between asphalt layers. PhD thesis, University of Nottingham. Disponível em $<$ http://eprints.nottingham.ac.uk/11115/> (acesso em 22/08/2019)

Uddim, W.; D. Zang e F. Fernandez (1994) Finite Element Simulation of Pavement Discontinuities and Dynamic Load Response. Transportation Research Record 1448. TRB, Washington, D.C., p. 100-106.*

Wu, S.; H. Chen; J. Zhang e Z. Zhang (2017) Effects of interlayer bonding conditions between semi-rigid base layer and asphalt layer on mechanical responses of asphalt pavement structure. International Journal of Pavement Research and Technology, 10(3), 274-281. doi:10.1016/j.ijprt.2017.02.003

Yassenn, O. M.; I. R. Endut; S. Z. Ishak; M. A. Hafez e Yaseen, H. M. (2015) Finite Element Modelling of Flexible Pavement. Journal of Multidisciplinary Engineering Science and Technology (JMEST) 3159-40, Vol. 2 Issue 1. Disponível em <http://www.jmest.org/wp-content/uploads/JMESTN42350341.pdf> (acesso em 22/08/2019)

Ziari H. e Khabiri M. M. (2007) Interface condition influence on prediction of flexible pavement life. Journal of Civil Engineering and Management. Vol XIII, No 1, p. 71-76. doi:10.3846/13923730.2007.9636421 